\title{
ADDENDUM: Direct brain infusion of glial cell line-derived neurotrophic factor in Parkinson disease
}

\section{S S Gill, N K Patel, G R Hotton, K O'Sullivan, R McCarter, M Bunnage, D J Brooks, C N Svendsen \& P Heywood Nat. Med. 9, 589-595 (2003)}

In response to a query on the limitations of presenting changes in drug treatment as percentage changes in levodopa equivalents, as shown in Table 1 of our article, we would like to submit further supplementary information detailing the actual drug changes during the course of this phase 1 clinical study. This information would allow for a better clinical interpretation of the participants, who showed both significant off- and on-medication benefit. Accordingly, we have tabulated the drug and levodopa equivalent changes up to 24 months, beyond the 12 -month clinical data and 18-month positron emission tomography data presented in our article.

\begin{tabular}{|c|c|c|c|c|c|c|}
\hline * & 666.7 & 666.7 & 666.7 & 733.3 & 733.3 & 733.3 \\
\hline P2 & $\begin{array}{l}\text { Sinemet CR QDS } \\
\text { Entacapone QDS }\end{array}$ & $\begin{array}{l}\text { Sinemet Plus } \times 5 / \text { day } \\
\text { Sinemet CR nocte } \\
\text { Tolcapone } 100 \mathrm{mg} \text { TDS }\end{array}$ & $\begin{array}{l}\text { Sinemet Plus } \times 5 / \text { day } \\
\text { Sinemet CR nocte } \\
\text { Tolcapone } 100 \text { mg TDS }\end{array}$ & $\begin{array}{l}\text { Sinemet Plus x 5/day } \\
\text { Sinemet CR nocte } \\
\text { Tolcapone } 100 \text { mg TDS }\end{array}$ & $\begin{array}{l}\text { Sinemet Plus x 5/day } \\
\text { Sinemet CR nocte } \\
\text { Tolcapone } 100 \text { mg TDS }\end{array}$ & $\begin{array}{l}\text { Sinemet Plus x 5/day } \\
\text { Sinemet CR nocte } \\
\text { Tolcapone } 100 \text { mg TDS }\end{array}$ \\
\hline * & 615.4 & 653.8 & 653.8 & 653.8 & 653.8 & 653.8 \\
\hline P3 & $\begin{array}{l}\text { Madopar } 125 \mathrm{mg} x \\
\text { 18-20/day } \\
\text { Sinemet Half CR x } \\
\text { 1-2/day } \\
\end{array}$ & $\begin{array}{l}\text { Cabergoline } 6 \text { mg/day } \\
\text { Sinemet Half CR x } \\
\text { 5-6/day }\end{array}$ & $\begin{array}{l}\text { Cabergoline } 6 \text { mg/day } \\
\text { Sinemet Half CR x 5-6/day }\end{array}$ & $\begin{array}{l}\text { Cabergoline } 6 \mathrm{mg} / \text { day } \\
\text { Sinemet Half CR } \times 4-5 / \text { day }\end{array}$ & $\begin{array}{l}\text { Cabergoline } 6 \text { mg/day } \\
\text { Sinemet Half CR x 4-5/day }\end{array}$ & $\begin{array}{l}\text { Cabergoline } 6 \text { mg/day } \\
\text { Sinemet Half CR } \times 5-6 / \\
\text { day }\end{array}$ \\
\hline * & 680 & 580 & 380 & 800 & 720 & 750 \\
\hline P5 & $\begin{array}{l}\text { Sinemet CR x 3/day } \\
\text { Sinemet Plus x 1/day } \\
\text { Cabergoline } 2 \text { mg/day } \\
\text { Zelapar } 1.25 \text { mg OD }\end{array}$ & $\begin{array}{l}\text { Cabergoline } 4 \mathrm{mg} / \text { day } \\
\text { Sinemet Plus x 3-4/day } \\
\text { Zelapar } 1.25 \mathrm{mg} \text { OD }\end{array}$ & $\begin{array}{l}\text { Cabergoline } 2 \text { mg/day } \\
\text { Sinemet Plus x 2.5-3/day } \\
\text { Zelapar } 1.25 \text { mg OD }\end{array}$ & $\begin{array}{l}\text { Cabergoline } 2 \text { mg/day } \\
\text { Sinemet Plus x 2.5/day } \\
\text { Zelapar } 1.25 \text { md OD }\end{array}$ & $\begin{array}{l}\text { Cabergoline } 3 \text { mg/day } \\
\text { Sinemet Plus x 2-2.5 }\end{array}$ & $\begin{array}{l}\text { Cabergoline } 2.5 \text { mg/day } \\
\text { Sinemet Plus x 1.75/day }\end{array}$ \\
\hline * & 761.5 & 800 & 500 & 450 & 500 & 425 \\
\hline
\end{tabular}

*Levodopa equivalents: $1 \mathrm{mg}$ Pergolide = $1 \mathrm{mg}$ lisuride $=10 \mathrm{mg}$ Bromocriptine = $10 \mathrm{mg}$ Apomorphine $=9 \mathrm{mg}$ Ropinirole = $1 \mathrm{mg}$ Cabergoline = $100 \mathrm{mg}$ Levodopa + dopa decarboxylase inhibitor $=130 \mathrm{mg}$ levodopa CR preparation. 